\title{
A desferoxamina e seu efeito protetor na preservação pulmonar
}

\author{
Luiz Sérgio FRAGOMENI*, Robert S. BONSER ${ }^{\star \star}$, Brian EDWARDS ${ }^{\star \star}$. Stuard W. JAMIESON**, Michael \\ P. KAYE**
}

RBCCV 44205-99

\begin{abstract}
FRAGOMENI, L. S.; BONSER, R. S.; EDWARDS, B.; JAMIESON, S. W.; KAYE, M. P. - Desferoxamina e seu efeito protetor na preservação pulmonar. Rev. Bras. Cir. Cardiovasc., 4(3): 237-241, 1989.

RESUMO: Há recentes evidências de que radicais livres derivados do oxigênio estão envolvidos na lesão tecidual decorrente de isquemia e subseqüente reperfusão. A desferoxamina (DF), evitando a produçāo de radicais hidroxila e eliminando o ânion superóxido, pode atenuar este dano, sendo seus efeitos aqui avaliados após quatro horas de preservação pulmonar hipotérmica. O auto-transplante pulmonar esquerdo foi realizado em 12 cães mestiços. O pulmão foi, inicialmente, perfurado com $1000 \mathrm{ml}$ de solução de Collins modificada e mantido insuflado, colocado sob refrigeraçāo $\left(4^{\circ} \mathrm{C}\right)$ em solução salina durante quatro horas. Seis cães receberam $500 \mathrm{mg}$ de DF administrados E.V. durante o período isquêmico e imediatamente após iniciar a reperfusāo. Após reimplante e ligadura da artéria pulmonar direita, os animais foram mantidos numa $\mathrm{FiO}_{2}$ fixa $(49 \%)$ e monitorizados por quatro horas. Durante a primeira hora pós-reperfusão, o $\mathrm{pO}_{2}$ arterial foi sifnificativamente superior no grupo tratado com DF $(p<0.05)$. $O$ gradiente alvéolo-arterial foi também inferior $(p<0.05)$. A resistēncia vascular pulmonar foi semelhante em ambos os grupos. Concluimos que a desferoxamina permite melhor troca gasosa no período imediatamente após reperfusão e que sua investigação, na área da preservação pulmonar, deve ser estimulada.
\end{abstract}

DESCRITORES: proteção pulmonar, desferoxamina; radicais livres.

\section{INTRODUÇÃO}

Durante os últimos anos, vários pesquisadores têm tentado explicar e relacionar as alteraçōes fisiopatológicas associadas com a reperfusão de tecido celular submetido a isquemia. Existem evidẽncias de que os radicais livres de oxigênio são, pelo menos em parte, responsáveis pelo dano celular decorrente da reoxigenação de tecidos que foram submetidos a hipóxia ${ }^{1}{ }^{12}$. 14. Órgãos como intestino delgado, estômago, fígado, pãncreas, músculo esquelético, rim e cérebro são afetados pela isquemia e subseqüente reperfusāo. Com o acúmulo de interesse no campo dos transplantes cardíaco e cardiopulmonar, as alteraçōes causadas por isquemia e reperfusão do miocárdio e tecido pulmonar têm sido, atualmente, mais intensamente investigadas ${ }^{2,7}$. 16. 18 .
Entre todos os radicais livres derivados do oxigênio envolvidos na lesão isquêmica e de reperfusão, acredita-se que o radical hidroxila $(-\mathrm{H})$ é o mais citotóxico, devido à sua capacidade de destruir biomoléculas, especialmente as membranas lipídicas ${ }^{9,12,17}$. A formação de maior quantidade de radicais hidroxila é proporcionado pela reação de Haber-Weiss, que, por sua vez, é catalizada pelo ion férrico ${ }^{5,9}$.

$$
\mathrm{O}_{2}^{-}+\mathrm{H}_{2} \mathrm{O}_{2} \longrightarrow \mathrm{Fe}^{3+} \mathrm{e} \longrightarrow \mathrm{O}_{2}+\mathrm{OH}^{-}+\mathrm{OH}
$$

Teoricamente, sem a presença de $\mathrm{Fe} 2$, esta reação é bem mais lenta e a formação do radical hidroxila será menos intensa.

Trabalho realizado no Minnesota Heart and Lung Institute. Minneapolis, Minnesota, USA.

Recebido para publicaçāo em 3 de novembro de 1990.

- Do Minnesota Heart and Lung Institute (Professor Convidado).

* Do Minnesota Heart and Lung Institute.

Endereço para separatas: Luiz Sérgio Fragomeni. Rua Uruguai, 1555, apt: 41. 96010 Passo Fundo, RS, Brasil. 
FRAGOMENI, L. S.; BONSER, R.; EDWARDS, B.; JAMIESON, S. W.; KAYE, M. P. - Desferoxamina e seu efeito protetor na preservação pulmonar. Rev. Bras. Cir. Cardiovasc., 4(3): 237-241, 1989.

A desferoxamina ${ }^{4}$, sendo um quelante do ion férrico, é uma potente inibidora do radical hidroxila durante períodos de isquemia e subseqüente reperfusão.

Baseados nessa hipótese, realizamos um modelo canino de auto-transplante pulmonar, no qual observamos os benefícios do quelante do ferro desferoxamina na prevenção de lesão isquemica e de reperfusão.

\section{MATERIAL E MÉTODO}

Doze cães mestiços pesando em média $27 \mathrm{k}$ foram anestesiados com tiamilal sódico (Biotal, 15-20 mg/k E. V.) e paralizados com suxametônio. O primeiro grupo de animais ( $n=6$ controle), após colocação de tubo endotraqueal, foram conectados ao respirador Servo 900 , com volume minuto de $20 \mathrm{ml} / \mathrm{k}$ e 12 movimentos respiratórios por minuto. A fração inspirada de $\mathrm{O}_{2}$ foi mantida a $40 \%$ com pressão expiratória positiva final de $5 \mathrm{~cm}$ de água, durante o experimento. Um cateter arterial $5 \mathrm{~F}$ para monitorização da pressão arterial foi colocado na carótida direita e um cateter de Swan-Ganz $7 F$, introduzido na veia jugular direita e orientado até o tronco pulmonar para medida de débito cardíaco (DC) pelo método de termodiluição. A pressão do átrio esquerdo foi medida através de cateter colocado sob visão direta.

O procedimento cirúrgico foi realizado através de toracotomia esquerda. Após dissecçāo do brônquio principal esquerdo, o pericárdio foi aberto anteriormente às veias pulmonares, expondo a artéria pulmonar esquerda. Após sua dissecçāo, a artéria pulmonar direita também foi exposta e reparada com fita umbelical, através do seio transverso, a fim de permitir o seu posterior pinçamento e ligadura. As veias pulmonares foram expostas, identificando-se sua comunicação com o átrio esquerdo.

Após heparinização sistêmica com 300 unidades de heparina $/ \mathbf{k}$, a artéria puomonar direita foi champeada e, após 10 minutos, os parâmetros de controle foram analizados, com o intuito de observar a performance do pulmão esquerdo antes de sua remoçāo e posterior reimplante. A seguir, após a liberaçāo da AP direita, o pulmão esquerdo foi removido após pinçamento da $\mathrm{AP}$, brônquio e átrio esquerdo, incluindo-se as respectivas veias pulmonares e o pulmāo removido com parte da parede atrial esquerda.

Em um recipiente com solução eletrolítica gelada, a artéria pulmonar foi perfundida com $1000 \mathrm{ml}$ de solução de Collins modificada a $4^{\circ} \mathrm{C}$, enquanto o pulmão era insuflado manualmente durante o período em que era perfundido. Após esse procedimento e submerso em solução eletrolítica a $4^{\circ} \mathrm{C}$, o pulmão foi deixado em refrigeração nessa temperatura durante 4 horas. Após esse período, o pulmāo foi re-implantado, anastomosando-se o átrio esquerdo com Prolene 5-0 e sutura contínua. O brônquio foi anastomosado com prolene 4-0 e, a seguir, a artéria pulmonar com Prolene 6-0. após terem sido permitidos 15 minutos de ventilação e reperfusão com o pulmão reimplantado, a artéria pulmonar direita foi ligada, a fim de manter o animal dependente do pulmão reimplantado. Após a monitorização por seis horas, o animal foi sacrificado, o pulmão esquerdo removido, pesado, e material retirado para avaliação anatomopatológica.

No segundo grupo de animais ( $n=6$ desferoxamina), o mesmo procedimento foi realizado, porém no período de uma hora e 30 minutos anteriormente à reperfusão pulmonar, $500 \mathrm{mg}$ de desferoxamina foram diluídos em $250 \mathrm{ml}$ de Ringer lactado e administrados E.V. durante período de duas horas.

Pressōes, DC, gases arteriais e eletrólitos foram analizados após o animal ter sido anestesiado, após o pinçamento da artéria pulmonar direita, após a remoçāo do pulmāo esquerdo e nos intervalos de 20,30 , $60,120,240$ e 360 minutos após o reimplante do pulmão esquerdo e subseqüente ligadura da artéria pulmonar direita.

O peso do pulmão reimplantado foi medido após a sua remoção e colocação em estufa a $45^{\circ} \mathrm{C}$ por três dias e o volume de retenção de líqüidos durante o experimento foi estimado. A resistência vascular pulmonar foi calculada usando-se os valores de DC, pressão média de artéria pulmonar e pressão de átrio esquerdo.

Todos os dados foram analisados através de teste T de Student, comparando a média dos grupos para significância das diferenças através dos valores de $P$. Valores de $P>0,05$ foram considerados sem diferença estatística.

\section{RESULTADOS}

Todos os dados coletados e a média dos grupos foram analisados estatisticamente para obter-se a signicicāncia das diferenças. A análise do sangue arterial durante as seis horas após o reimplante demonstrou melhor oxigenação do grupo tratado com desferoxamina, com significativa diferença nos primeiros 20 minutos após o reimplante $(P<0,05) 30$ minutos $(P<0,02)$ e 60 minutos $(P<0,05)$ (Figura 1). Embora sem diferença estatística, a oxigenaçāo permanece superior nos outros intervalos. O gradiente arterial/alveolar também demonstrou alteraçōes semelhantes com gradientes inferiores no grupo tratado com desferoxamina e valores de $P$ significativos nos intervalor de 20 minutos $(P<0,01)$, 30 minutos $(P<0,02)$ e 60 minutos $(P<0,05)$ que sucederam à reperfusão (Figura 2). $\mathrm{O}$ peso pulmonar seco também foi inferior no grupo tratado; porém, sem significãncia estatística $(.10<p<.20)$ (Figura 3). A histopatologia de duas diferentes áreas em cada pulmão transplantado demonstrou graus variáveis de edema alveolar, edema intersticial, pneumonite ou pleurite em ambos os grupos; porém, estas alteraçōes nunca foram significativas. Todos os outros dados: resistência arterial 


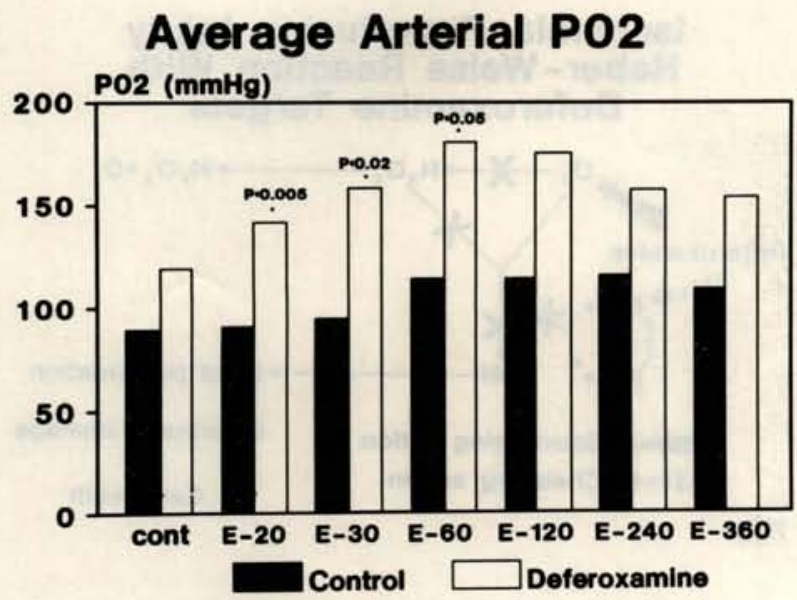

Fig. 1

\section{A-a Gradients}

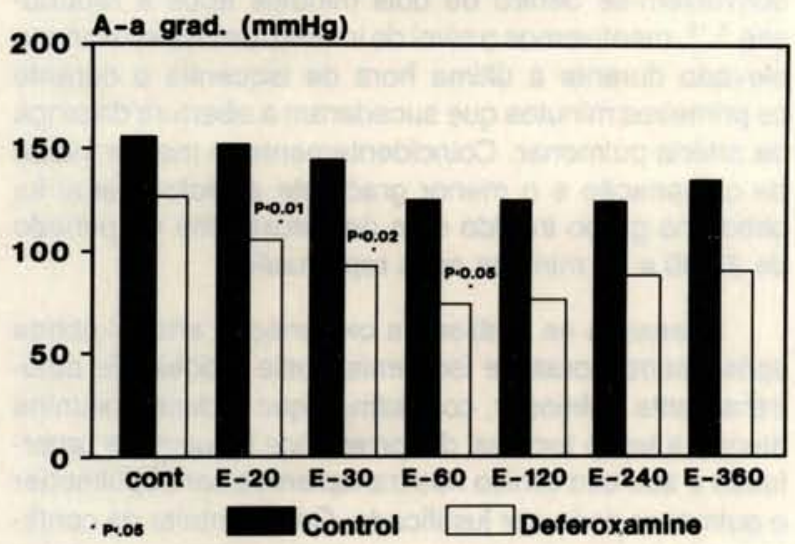

Fig. 2

\section{Lung Weights}

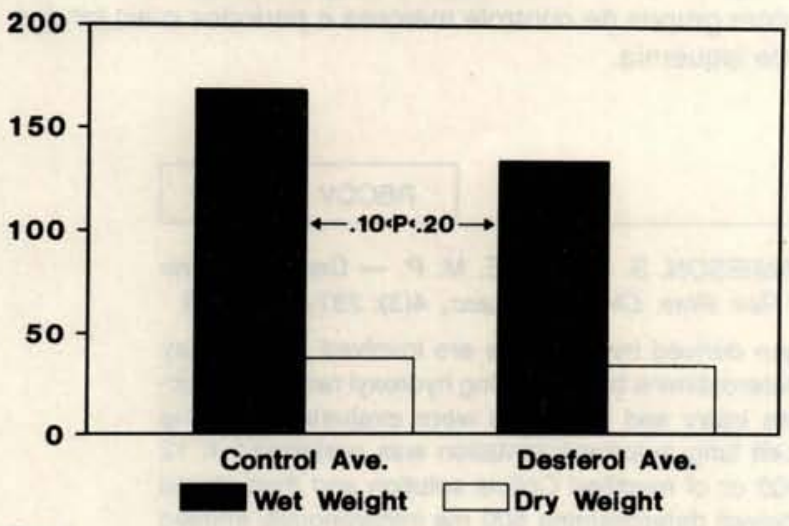

Fig. 3

pulmonar (PVR, Figura 4), ${ }_{\mathrm{p}} \mathrm{CO}_{2}$ (Figura 5), eletrólitos e DC mantiveram-se semelhantes nos dois grupos estudados.

O progresso técnico alcançado com o transplante cardiopulmonar e pulmonar tem estimulado a investi-

\section{Average PVR}

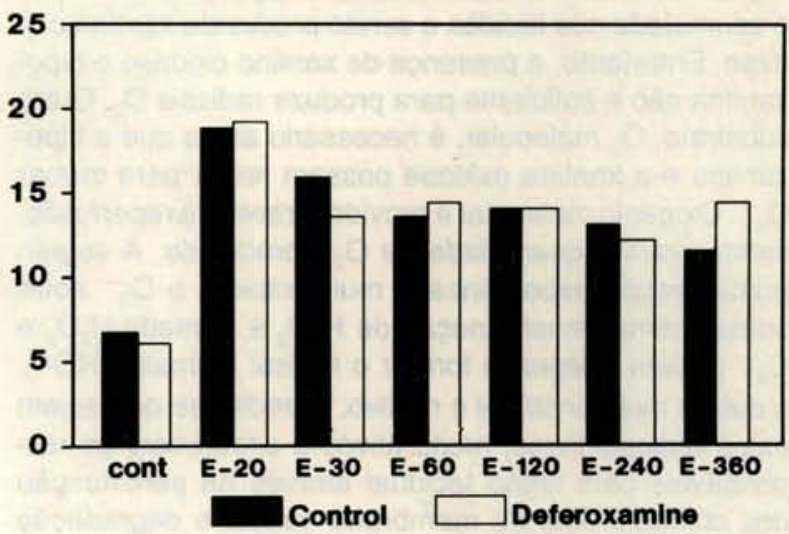

Fig. 4

\section{Average Blood PCO2}

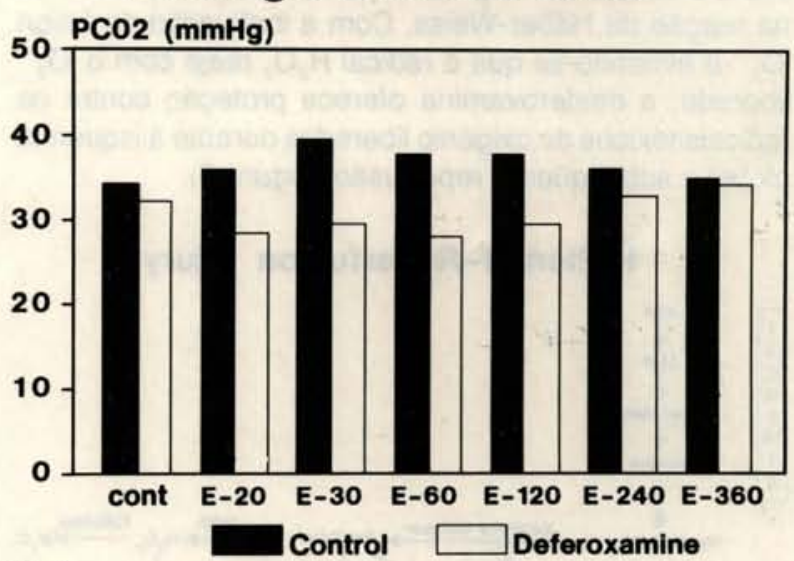

Fig. 5

gação de formas mais satisfatórias de proteção do parênquima pulmonar à lesão isquêmica e de reperfusão.

Apesar dos métodos de preservação miocárdica estarem, hoje, bem estabelecidos, permitindo clinicamente ótima função miocárdica após períodos de quatro horas e meia de isquemia, o tecido pulmonar, devido às suas características fisiológicas, ainda é mais suscetivel a esses procedimentos. O leito capilar e os septos alveolares são mais sensiveis à lesão isquêmica, resultando em edema, rotura da membrana alvéolo-capilar e subse-

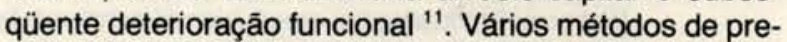
servaçāo tecidual contra a isquemia, bem como a adição de drogas (SOD, DMSO, catalase. alopurinol) que possam auxiliar na prevenção da formação de radicais $\mathrm{HO}$ • tóxicos ao tecido celular têm sido investigados ${ }^{2,} 6,13$, 16, 19, 20 . Apesar de a desferoxamina já ter sido investigada na prevenção da lesão de reperfusão no miocárdio, seus benefícios foram, aqui, pela primeira vez experimentalmente evidenciados na preservaçāo do tecido pulmonar.

O esquema proposto por GRANGER et alii ${ }^{10}$, explicando o mecanismo de formação do radical $\mathrm{O}_{2}$ pós isque- 
FRAGOMENI, L. S.; BONSER, R.; EDWARDS, B.; JAMIESON, S. W.; KAYE, M. P. - Desferoxamina e seu efeito protetor na preservação pulmonar. Rev. Bras. Cir. Cardiovasc., 4(3): 237-241, 1989.

mia, é demonstrado na Figura 6. Durante o período de isquemia, o ATP é catabolizado à hipoxantina, a qual é acumulada nos tecidos e sendo produzida xantina oxidase. Entretanto, a presença de xantina oxidase e hipoxantina não é suficiente para produzir radicais $\mathrm{O}_{2}$. Outro substrato, $\mathrm{O}_{2}$ molecular, é necessário antes que a hipoxantina e a xantina oxidase possam reagir para formar $\mathrm{O}_{2}^{-}$. Oxigênio molecular é provido através da reperfusão, sendo grande quantidade de $\mathrm{O}_{2}^{-}$produzido. A seguir, numa reaçāo espontânea e muito rápida, $\mathrm{O}_{2}^{-}$sofre subseqüente transformação de $\mathrm{H}_{2} \mathrm{O}_{2}$ é formado. $\mathrm{H}_{2} \mathrm{O}_{2}$ e $\mathrm{O}_{2}^{-}$podem integrar e formar o radical hidroxila $\left(\mathrm{HO}^{\circ}\right)$, o qual é muito instável e reativo, Acredita-se que sejam estes radicais livres, muito ativos e citotóxicos, os responsáveis pela lesāo tecidual através da peroxidação dos componentes da membrana celular e degradação do ácido hialurônico e do colágeno ${ }^{8}$.

Neste estudo, idealizamos utilizar a capacidade da desferoxamina em suprimir o poder catalizador do ferro na reação de Haber-Weiss. Com a inativação do ânion $\mathrm{O}_{2}-$ e evitando-se que o radical $\mathrm{H}_{2} \mathrm{O}_{2}$ reaja com o $\mathrm{O}_{2}^{-}$ liberado, a desferoxamina oferece proteção contra os radicais tóxicos de oxigênio liberados durante a isquemia global e subseqüente reperfusão (Figura 7).

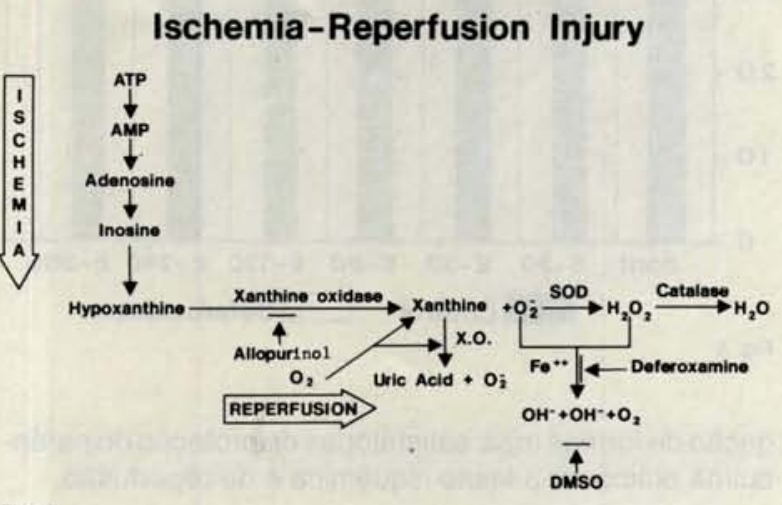

Fig. 6

\section{Ischemia-Reperfusion Injury Haber-Weiss Reaction With Deferoxamine Targets}

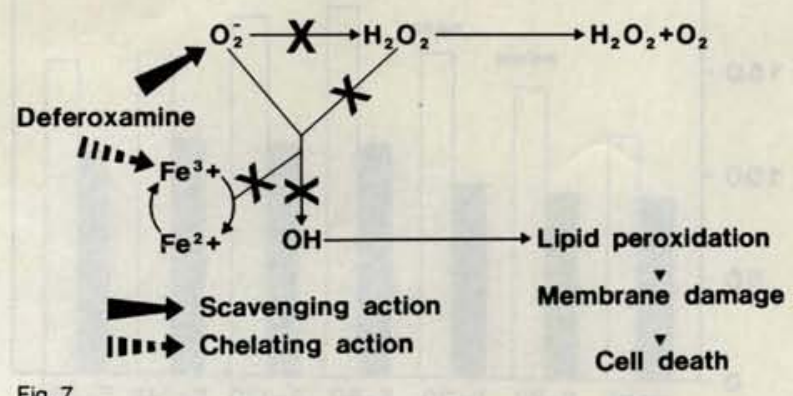

Fig. 7

Com a suposição de que a concentração de radicais livres de $\mathrm{O}_{2}$ é alcançada em 30 segundos e que as alteraçōes decorrentes da recuperação metabólica desenvolvem-se dentro de dois minutos após a reperfusão ${ }^{3,15}$, mantivemos o nível de infusāo de desferoxamina elevado durante a última hora de isquemia e durante os primeiros minutos que sucederam a abertura da pinça da artéria pulmonar. Coincidentemente, o melhor indice de oxigenação e o menor gradiente alvéolo-arterial foi obtido no grupo tratado com desferoxamina no período de 20-30 e 60 minutos após reperfusão.

Baseados na análise da oxigenação arterial obtida após quatro horas de isquemia neste modelo de autotransplante pulmonar, concluímos que a desferoxamina atenua a lesão tecidual decorrente de isquemia e reperfusão e seu uso clínico nos transplantes cardiopulmonar e pulmonar pode ser justificado. Com o intuito de confirmarmos esses promissores resultados iniciais, presentemente continuamos investigando os efeitos que a combinaçāo de drogas eliminadoras de radicais tóxicos de oxigênio possa ter na área da preservação pulmonar, com grupos de controle maiores e períodos mais longos de isquemia.

\section{RBCCV 44205-99}

FRAGOMENI, L. S.; BONSER, R. S.; EDWARDS, B.; JAMIESON, S. W.; KAYE, M. P. - Desferoxamine and its protective effect in pulmonary preservation. Rev. Bras. Cir. Cardiovasc., 4(3): 237-241, 1989.

ABSTRACT: There is increasing evidence that oxygen derived free radicals are involved in the injury that occurs following the reperfusion of ischaemic tissue. Deferoxamine by preventing hydroxyl radical production and scavenging superoxide anion may attenuate this injury and its effects were evaluated following 4 hours of static hypothermic pulmonary preservation. Left lung autotransplantation was performed in 12 mongrel dogs. The autograft was initially flushed with $1000 \mathrm{cc}$ of modified Collins solution and then stored in an inflated state for 4 hours in $4^{\circ} \mathrm{C}$ saline. Six dogs received deferoxamine $500 \mathrm{mg}$ intravenously infused before and immediately after the time of reperfusion. Following reimplantation, contralateral pulmonary artery ligation was performed and the animals were then maintained on a fixed $\mathrm{FiO}_{2}(40 \%)$ and monitored for 6 hours. During the first hour of reperfusion arterial $\mathrm{pO}_{2}$ was significantly better in the deferoxamine treated dogs $(p<0.05)$. Alveolar-arterial oxygen gradients were also lower $(p<0.05)$. Pulmonary vascular resistance was not different between groups. We conclude that deferoxamine improves gas exchange in the immediate reperfusion period and that further investigation of its use is warranted.

DESCRIPTORS: pulmonary preservation, deferoxamine; free radicals. 
FRAGOMENI, L. S.; BONSER, R.; EDWARDS, B.; JAMIESON, S. W.; KAYE, M. P. - Desferoxamina e seu efeito protetor na preservaçāo pulmonar. Rev. Bras. Cir. Cardiovasc., 4(3): 237-241, 1989.

\section{REFERÊNCIAS BIBLIOGRÁFICAS}

1 AMBROSIO, G.; WEISFELDT, M. L.; JACOBUS, W. E.; FLAHERTY, J. T. - Evidence for a reversible, oxygen radical mediated component of repertusion damage: reduction by recombinant human superoxide dismutase administered at the time of reflow. Circulation. 75: 282-291, 1987

2 AMBROSIO, G.; ZWEIER, J. L.; JACOBUS, W. E.; WEISFELDT, M. L.; FLAHERTY, J. T. - Improvement of postischemic myocardial function and metabolism induced by administration of deferoxamine at the time of reflow: the role of iron in the pathogenesis of reperfusion injury. Circulation, 76: 906-915, 1987.

3 AMBROSIO, G.; ZWEIER, J. L.; WEISFELDT, M. L.; FLAHERTY, J. T. - Early toxic effects of oxygen free radicals on myocardial energy metabolism following reperfusion. J. Am. Coll. Cardiol., 9: 125A, 1987.

4 AUST, S. D.; MOREHOUSE, L. A.; THOMAS, C. E. Role of metals in oxygen radical ractions. J. Free Radicals Biol. Med., 1: 3-25, 1985.

5 AUST, S. D. \& WHITE, B. C. - Iron chelation prevents tissue injury following ischemia. Adv. Free Radicals Biol., 1: 1-17, 1985.

6 BANDO, K.; TAGO, M.; TERAMOTO, S. - Prevention of free radical-induced myocardial injury by allopurinol. J. Thorac. Cardiovasc. Surg., 95: 465-473, 1988.

7 CASALE, A. A.; BUKLEY, G. B.; BULKLEY, B. H.; FLAHERTY, J. T.; GOTT, V. L.; GARDNER, T. J. - Oxygen free-radical scavengers protect the arrested, globally ischemic heart upon reperfusion. Surg. Forum, 34: 313-316, 1983.

8 FRIDOVICH, 1- The biology of oxygen radicals. Science, 210: 875-880, 1978.

9 GRAF, E.; MAHONEY, J. R.; BRYANT, R. G.; EATON, J. W. - Iron-catalyzed hydroxyl radical formation: stringent requirement for free iron coordination site. $J$. Biol. Chem., 259: 3620-3624, 1987.

10 GRANGeR, D. N.; HOLLWARTH, M. A.; PARKS, D. A. - Ischemia reperfusion injury: role of oxygen-derived free radicals. Acta Physiol. Scand., 126 (Supl. 1): $47-63,1986$
11 HAVERICH, A.; SCOTT, W. C.; JAMIESON, S. W. Twenty years of lung preservation: a review. Heart Tranplant., 4: 234-240, 1985.

12 HESS, M. L. \& MANSON, N. H. - Molecular oxygen: friend and foe. The role of the oxygen free radical system in the calcium paradox, the oxygen paradox and ischemia/reperfusion injury. J. Mol. Cell. Cardiol., 16: 969-985, 1984.

13 JURMANN, M. J.; SCHAEFERS, H. J.; DAMMENHAYN, L.; HAVERICH, A. - Oxygen-derived free radical scavengers for amelioration of reperfusion damage in heart transplantation. J. Thorac. Cardiovasc. Surg., 95: 368-377, 1988.

14 McCORD, J. M. - Oxygen-devised free radicals in post-ischemic tissue injury. $\quad$ N. Engl. J. Med., 312: 159-163, 1985.

15 McCORD, J. M. \& FRIDOVICH, I. - Superoxide dismutase: an enzymic function for erythrocuprein (hemocuprein). J. Biol. Chem., 244: 6049-6065, 1969.

16 MENASCHE, P.; GROUSSET, C.; GAUDUEL, Y.; MOVAS, C.; PIWNICA, A. - Prevention of hydroxyl radical formation. A critical concept for improving cardioplegia: protective effects of deferoxamine. Circulation, 76 (Supl. 5): 180-185, 1988.

17 MYERS, C. L.; WEISS, S. J.; KIRSH, M. M.; SHLAFER, M. - Involvement of hydrogen peroxide and hydroxyl radical in the "oxygen paradox" rduction of creatine kinase release by catelase, allopurinol or deferoxamine but not by superoxide dismutase. J. Moll. Cell Cardiol., 17: 675-684, 1985.

18 SHLAFER, M.; KANE, P. F.; WIGGINS, V. Y.; KIRSH, M. M. - Possible role for cytotoxic oxygen metabolites in the pathogenesis of cardiac ischemic injury. Circulation., 66 (Supl. 1): 85-92, 1982.

19 SIMPSON, P.; MICKELSON, J. K.; LUCCHESI, B. R. Free radical scavengers in myocardial ischemia. Fed. Proc., 46: 2413-2421, 1987.

AGRADECIMENTO: Este trabalho não poderia ter sido completado sem o inestimável auxílio dos Técnicos em Laboratório Edward Van Bergen e Sally Brown. 